Economía, Sociedad y Territorio, vol. vi, núm. 23, 2007, 693-724

\title{
Estructura y eficiencia urbanas. Accesibilidad a empleos, localización residencial e ingreso en la zMCM 1990-2000
}

\author{
Manuel Suárez-Lastra* \\ Javier Delgado-Campos**
}

\begin{abstract}
The sub-urbanisation of employment has lead to the thought that the urban structure in the city is changing from being mono-centred to becoming poly-centred and thus will tend to be more efficient. On the contrary, through gravitational analysis of accessibility, in this study we propose that this supposition is false and that in the last decade the zones with greater population growth have lost accessibility to employment. In the same manner, the preliminary results of measuring the employment accessibility indicate that the population with lower income are the least favoured. Finally, we present some evidence that suggests the need of urban planning based on employment accessibility as well as some recommendations for urban public policy.
\end{abstract}

Keywords: accessibility, transport, urban planning, spatial segregation, sub-urbanisation

\section{Resumen}

La suburbanización del empleo ha llevado a pensar que la estructura urbana de la ciudad está cambiando de monocéntrica hacia una policéntrica y, por lo tanto, tenderá a ser más eficiente. Por el contrario, en este estudio proponemos, mediante análisis gravitacionales de accesibilidad a empleos, que este supuesto es falso y, más aun, que las zonas de mayor crecimiento poblacional han perdido accesibilidad a empleos en la última década. Asimismo, los primeros resultados de medir la accesibilidad a empleos indican que la población en los estratos de ingreso más bajos son los más desfavorecidos. Finalmente, presentamos la evidencia que sugiere la necesidad de planeación urbana con base en accesibilidad a empleos, así como una serie de recomendaciones de política pública urbana.

Palabras clave: accesibilidad, transporte, estructura urbana, segregación espacial, suburbanización.

*Instituto de Geografía de la Universidad Nacional Autónoma de México. Correo-e: elmanix@gmail.com,msuarezl@prodigy.net.mx

*:Instituto de Geografía de la Universidad Nacional Autónoma de México. Correo-e: jdelgado@igiris.igeograf.unam.mx 


\section{Presentación}

Tiene lugar un debate acerca de si la Ciudad de México es o no una metrópoli policéntrica. Es un hecho que la proporción de empleos que las cuatro delegaciones centrales aportan a la Zona Metropolitana de la Ciudad de México (ZMcm) ha disminuido considerablemente en las últimas décadas. Pero aun teniendo en cuenta esta disminución proporcional, tres de ellas se colocaron entre las cuatro delegaciones con el mayor crecimiento absoluto de empleos en la ZMCM entre 1990 y 2000.

La disyuntiva entre considerar la proporción de crecimiento o bien el crecimiento absoluto de empleos con relación a su localización y tipo, genera controversias sobre la primacía de la ciudad interior y da pie a tres hipótesis distintas acerca de la transformación de la estructura del empleo urbano en la ciudad: monocentrismo con dispersión, policentrismo y desarrollo en corredores (strip development).

Los tres casos suponen la pérdida de primacía del centro original. En el primer caso, el centro tradicional se extiende sobre una superficie cada vez mayor pero a menor densidad conforme aumenta la distancia al mismo, dejando de ser un centro compacto. En el segundo, se crean nuevos subcentros separados físicamente del centro original, y en el tercer caso, la estructura central se mantiene, pero se desarrollan zonas de empleo a lo largo de vías de transporte que emanan del centro original.

El hecho es que hoy en día los especialistas sobre el tema aseguran que la teoría urbana clásica basada en el modelo monocéntrico ya no explica la estructura de las grandes ciudades. Y es que con el crecimiento, esta estructura monocéntrica deja de ser funcional. La consecuencia es una fase de transición hacia una estructura urbana alternativa más eficiente (Aguilar y Alvarado, 2005). ${ }^{1}$

En el presente trabajo se mide la eficiencia del incremento y localización de los mercados de empleo en la ZMCM en relación con la localización de las zonas residenciales por medio de índices de accesibilidad a empleos entre 1990 y 2000. La discusión se centra en determinar el tipo de transformación que la ciudad ha tenido y la calidad de la misma.

La conclusión es que, cualquiera que sea la forma urbana hacia la que transita la ZMCM, la accesibilidad a empleos ha disminuido en la periferia de la ciudad interior y en las zonas de mayor

\footnotetext{
${ }^{1}$ Para un primer acercamiento a este debate, véase Suárez y Delgado (en prensa).
} 
crecimiento demográfico, a pesar de su incremento proporcional de empleos. Por el contrario, quienes viven cerca de la ciudad interior tienen mayores oportunidades de empleo apropiados a los sectores de ocupación y categorías de ingreso de la población de estas áreas y, por lo tanto, existe un "desequilibrio espacial” (spatial mismatch) generalizado que se acentúa en el tiempo, con relación a la distancia al centro y, por ende, con relación al crecimiento de la ciudad.

El artículo está dividido en tres secciones. En la primera se hace un recuento de la teoría y las discusiones más trascendentes respecto de la accesibilidad, el desequilibrio espacial y el equilibrio entre empleo y vivienda (jobs-housing balance). En la segunda parte se presentarán la metodología empleada en el estudio y los resultados del análisis. Posteriormente, en la tercera sección se presentan una serie de conclusiones y recomendaciones de política pública.

\section{Teoría e investigaciones previas}

El término accesibilidad, con frecuencia, es confundido con el de movilidad, aunque, sin duda, está relacionado con él. En su significado más simple, la accesibilidad es la oportunidad de llegar a un lugar útil desde otro. Una carretera de seis carriles en medio de un desierto tendría un alto índice de movilidad por la velocidad que un automóvil podría alcanzar en ella; sin embargo, si en los extremos de la carretera no existiera nada, la accesibilidad que ésta produciría sería nula.

La accesibilidad tiene, por lo tanto, dos componentes: un componente de transporte (o factor de resistencia) y un componente de actividad (o factor de motivación) (Handy, 1993). Estos componentes obligan al estudio de los usos del suelo y de la concentración de actividades económicas y residenciales con relación a los medios de transporte que las conectan tomando en consideración la distancia entre ellas. Visto así, estudiar la accesibilidad significa evaluar la eficiencia de la estructura urbana con relación a su organización socioeconómica-espacial interna.

Las medidas de accesibilidad a empleos tienen origen en los estudios sobre la segregación racial y de ingreso en Estados Unidos de América. En 1964, John Kain propuso la hipótesis del desequilibrio espacial. En 1968, en "Housing Segregation, Negro Employment, and Metropolitan Decentralization”, aportó la evidencia empírica que probaba que las limitaciones en la elec- 
ción del lugar de residencia de los afro-americanos en Estados Unidos, junto con la dispersión de empleos de las ciudades centrales, eran los factores causales de las bajas tasas de empleo y bajo ingreso (Kain, 1994). El estudio se basaba en la evidente transformación hacia estructuras policéntricas de las ciudades norteamericanas como resultado de la suburbanización del empleo. Por su parte, Mills (1972) demostró que la baja accesibilidad a empleos que caracterizaba a la población negra no se debía a una cuestión de raza sino a una de educación e ingreso. Dado que la curva de oferta de renta (bid-rent curve) de los pobres tiene una pendiente mayor, éstos tienden a vivir en el centro. Con la suburbanización del empleo, se crea un patrón de desequilibrio espacial entre los ocupantes de las viviendas de bajos ingresos y los empleos que pueden obtener. De acuerdo con la lógica de la teoría urbana clásica de localización económica (Alonso, 1964), esto se debe a que los primeros empleos en suburbanizarse son aquellos que tienen el menor valor agregado de producción y los que requieren una menor calificación. De esta forma, el desequilibrio espacial afectaría a hogares tanto blancos como negros en condiciones de pobreza. Este punto es fundamental pues, en el caso de la Ciudad de México, la raza no es un factor pertinente, pero sí lo es la categoría de ingreso.

Arnott (1997) clasifica los enfoques sobre el desempate espacial en dos tipos. Un primer enfoque subraya la oportunidad de obtener un empleo mediante el conocimiento de la oferta en redes de información (Holzer et al., 1994; O’Regan y Quigley, 1993). Este enfoque subraya que la información sobre empleos suburbanos es difícil de obtener en el centro de la ciudad, más aún si ésta fluye mediante un sistema de persona a persona (mouth-to-mouth) y depende de que el interesado tenga conocidos y conexiones para obtener un empleo (Chapple, 2001).

Un segundo enfoque centra la discusión de la baja oportunidad de empleos para los habitantes de la ciudad central en los costos del traslado (commuting costs) (Cervero et al., 1997, Ihlanfeldt y Sjoquist, 1989). Si debido a la suburbanización de los empleos, la posibilidad de obtener uno disminuye para quienes viven en la ciudad interior, estos residentes tendrán que realizar viajes más largos al trabajo o bien quedar desempleados. Los costos del traslado se incrementan, pues el transporte público hacia los suburbios suele ser de baja calidad, la densidad de servicio es poca y el servicio no es frecuente. En el presente análisis se adopta esta segunda perspectiva, pero aplicada a la ZMCM. 
Cervero es uno de los precursores en la medición del desequilibrio espacial con base en índices gravitacionales de accesibilidad, entendida ésta como el número de oportunidades reales de trabajo que tienen los pobladores de una zona determinada, tomando en cuenta la distancia entre las zonas residenciales y los centros de empleos y emparejando empleos y trabajadores de acuerdo con sectores económicos (Cervero, 1997; Cervero et al., 1997). En diversos análisis sobre el Área de la Bahía de San Francisco, Cervero ha insistido en que la principal causa del desequilibrio espacial es la falta de un balance entre empleos y vivienda (jobs-housing balance) (Cervero, 1989, Cervero, 1995, Cervero, 1996). Esta propuesta ha generado una gran discusión entre académicos, en favor y en contra.

$Y$ es que la idea de accesibilidad entre empleos y residencia supone que la ciudad está estructurada alrededor de los mercados de trabajo y que, por lo tanto, la localización residencial debe estar ligada a la ocupación de quienes trabajan. Esta idea es, hasta cierto punto, la continuación de una larga tradición entre economistas, aunque, frecuentemente, de diferentes corrientes, que reflejan la importancia de las estructuras laborales y económicas en la organización social y datan desde Smith, pasando por Marx, Marshall, Polanyi y Alonso.

A Guliano y Small (1993) se debe la principal crítica a la propuesta del equilibrio entre vivienda y empleo. Argumentan que dicho desequilibrio no afecta sustancialmente los tiempos de recorrido al trabajo (aunque es estadísticamente significativo) y que, por lo tanto, las políticas metropolitanas que busquen alterar la estructura del suelo urbano están destinadas al fracaso. En su opinión, el tiempo de recorrido al trabajo pudiera servir como amortiguador (buffer) psicológico entre el hogar y el empleo. Asimismo, la existencia cada vez mayor de hogares con más de un trabajador hace imposible que una residencia se localice cerca de los empleos de todos sus miembros trabajadores. Sin embargo, en su propio modelo han logrado explicar cerca de cincuenta por ciento del tiempo promedio de traslado mediante un modelo de programación lineal de "traslado en exceso" (excess commute) tomando en cuenta sectores económicos de ocupación pero sin contar categorías de ingreso entre residentes y empleos. ${ }^{2}$

\footnotetext{
${ }^{2}$ Para una aplicación de este método a la ZMCM, véase Murata, Suárez y Delgado (2005).
} 
Finalmente, Giuliano y Small (1993) niegan que exista tal desequilibrio espacial, e instan a que se deje actuar al mercado y a que, por medio de él se alcance la eficiencia de usos de suelo. Sin duda, ésta es una visión que le concede demasiados méritos al mercado, al grado de extenderle el nivel de equilibrador de la segregación social.

\subsection{Aplicabilidad de la perspectiva analítica norteamericana a la realidad de la ZMCM, 1990-2000}

No existe, hasta el día de hoy, un estudio sistemático sobre la accesibilidad a empleos en la ZMCM. Hasta hace poco tiempo, las investigaciones que aludían a la idea de accesibilidad se habían limitado a hablar sobre la relación centro-periferia entre empleo y vivienda, y sobre la desdensificación y terciarización del centro, o bien habían descrito los flujos de transporte en la zona metropolitana (Cruz Rodríguez, 2001; Delgado et al., 1999; Villavicencio; 1999). Asimismo, se han realizado diversos estudios descriptivos sobre las formas y flujos de transporte basados en encuestas de origen-destino de la Ciudad de México y limitados, en su mayoría, a la descripción de la deficiencia de la estructura de trasporte dadas las distancias y tiempos de recorrido, y de la movilidad de la fuerza laboral de la ciudad (Lizt, 1988; Muñoz, 1995; Navarro, 1988, Quintanilla; 1995). Aunque existe una relación necesaria entre uso del suelo y transporte, pocos investigadores dedicados al transporte ponen atención al uso del suelo. Por su parte, quienes investigan sobre el uso del suelo y la actividad económica, en particular quienes estudian la vivienda, se basan en generalizaciones sobre el transporte o bien no hacen ninguna mención. Si bien estos estudios han sido importantes para la comprensión de los fenómenos metropolitanos, son insuficientes.

Estudios más recientes, como los de Grazibord y Acuña (2005) y Aguilar y Alvarado (2005), partiendo de distintos enfoques metodológicos han sugerido que la estructura monocéntrica de la ZMCM está cambiando a una estructura policéntrica (multinodal o polinuclear, en sus propios términos). Otros autores han definido a la ciudad con una estructura de "policentrismo [y] centralidades que carecen de autosuficiencia socioeconómica [que] mantienen una relación de dependencia con el núcleo central de la ciudad, que conserva así su capacidad altamente centralizadora y concentradora de actividades, funciones y recursos" (Delgado et al., 1999). 
La base común tácita de los estudios citados, aunque sigan perspectivas distintas (flujos de transporte, forma urbana y crecimiento socioeconómico), es la relación entre el uso del suelo y el transporte. El uso de suelo se refiere a las actividades que se realizan en un determinado espacio, mientras que por el transporte se entiende la relación socioeconómica y espacial entre los distintos espacios. Por su parte, el crecimiento socioeconómico se refiere a cómo se transforman los espacios existentes y cómo se estructuran los nuevos espacios urbanos.

Las conclusiones que se desprenden de estos estudios son en momentos coincidentes y en momentos opuestas. Aunque en ninguno se niega que exista una suburbanización del empleo (esto lo indican las estadísticas), unos suponen un centro cada vez más fuerte con una periferia desarticulada y con empleos dispersos que genera una acentuada segregación social, y, otros, una estructura que tiende a formar subcentros (sin aceptar o rechazar que sea más eficiente).

En la perspectiva aquí propuesta, es decir, en términos de accesibilidad a empleos, el supuesto inicial es que la suburbanización económica genera mayores oportunidades de empleo en la periferia y, por lo tanto, mayor accesibilidad. Pero cacaso esto es cierto? Dependiendo de las estadísticas que se analicen, esto podría o no ser así.

En las figuras i y II se muestran dos perspectivas. En la primera se muestra el crecimiento de empleos y Población Económicamente Activa (PEA) por contorno urbano, y en la segunda, la proporción crecimiento metropolitano de cada contorno entre 1990 y 2000. El primer resultado interesante es que a pesar del mencionado despoblamiento de la ciudad interior (-12.3\% entre 1990 y 2000) la PEA y la PEA ocupada tuvieron un ligero incremento en la ciudad interior (0.8 y 1.5\%, respectivamente). Ello habla, hasta cierto punto, de la movilidad residencial dentro de la ZMCM y, mejor aún, de las características de quienes llegan y de quienes se van. El segundo resultado pertinente es que la diferencia entre las proporciones de crecimiento entre PEA es mayor en la ciudad interior y en el cuarto contorno que en los contornos intermedios (véase figura I).

Sin embargo, si se compara lo anterior con los datos de la figura II, el resultado se vuelve alarmante. Excepto en el primer y cuarto contornos, donde la aportación del crecimiento a la ZMCM es más o menos equilibrado, el mayor aumento de empleos sucede en el centro y decrece con la distancia, mientras que el mayor 


\section{Figura I}

Proporción de crecimiento de PEA y empleos por contorno metropolitano 1990-2000

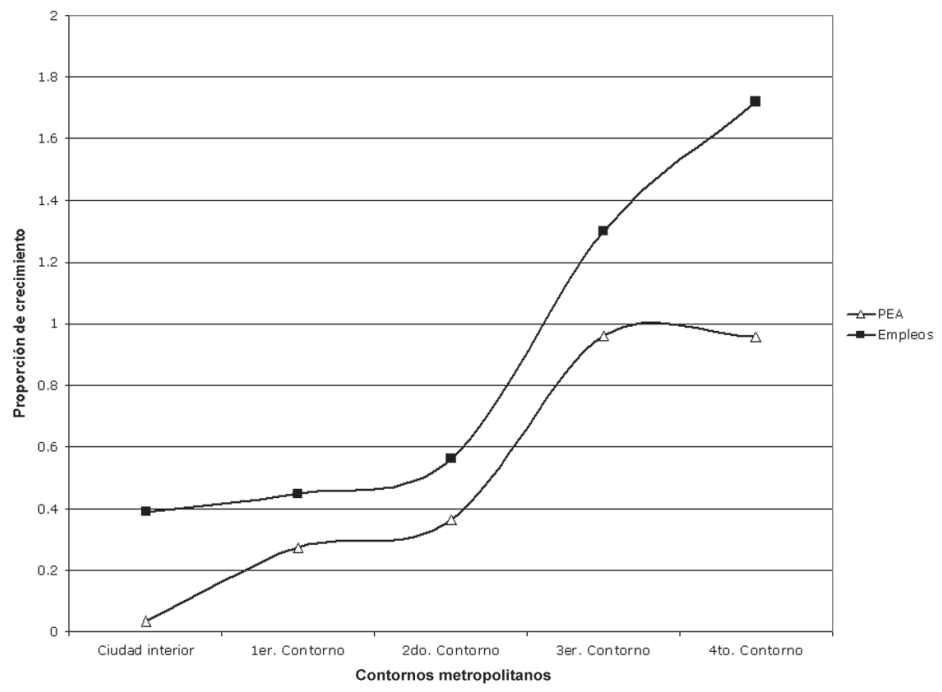

Fuente: Elaboración propia con datos de los Censos Económicos 1989 y 1999 y de los Censos Generales de Población y Vivienda 1990 y 2000.

\section{Figura II}

\section{Proporción del crecimiento total de empleos y PEA por contorno metropolitano}

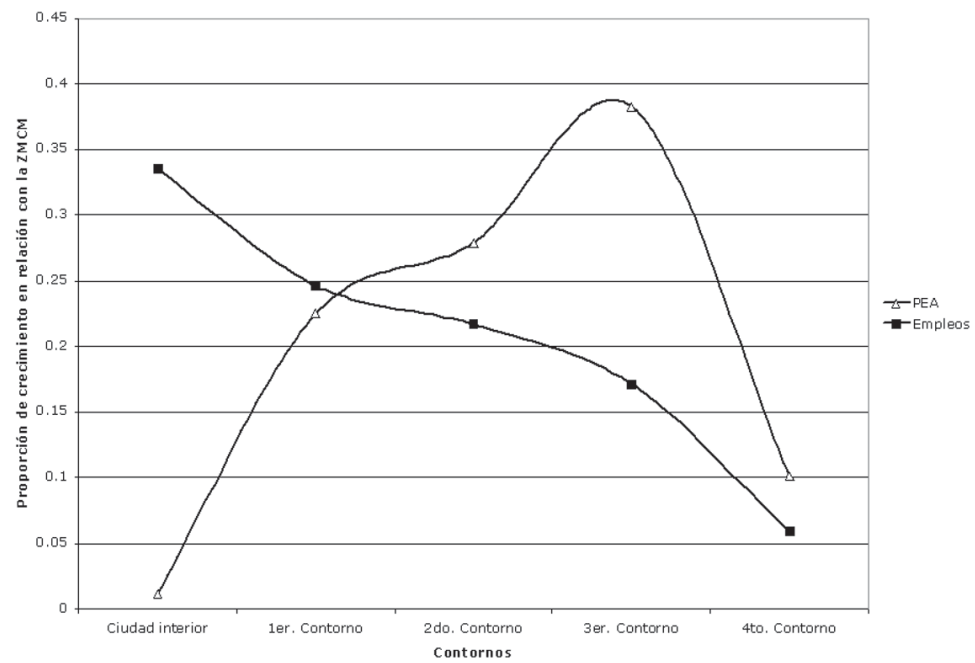

Fuente: Elaboración propia con datos de los Censos Económicos 1989 y 1999 y de los Censos Generales de Población y Vivienda 1990 y 2000. 
crecimiento de la PEA se registra en el segundo y tercer contornos. ¿Significa esto que la accesibilidad a empleos ha sido mayor en el centro y en la periferia de la ciudad? ¿Ha sido eficiente la generación de empleos en la ZMCM en términos espaciales? ¿Puede suponerse que se están formando subcentros? ¿O es que la suburbanización de empleos ha tomado, por el momento, la forma de dispersión (sprawl) económica? A continuación se muestra un análisis detallado de accesibilidad para la ZMсм que ayudará a resolver estos cuestionamientos.

\section{Evaluación de la accesibilidad a empleos}

Para medir los cambios en la accesibilidad a empleos en la ZMCM se utilizaron varios índices que miden la oportunidad de empleo que tienen los trabajadores de los distintos municipios de la ciudad, tomando en cuenta la distancia como elemento de fricción. Dada la creciente actividad económica periférica, se consideraron todos los empleos por municipio de la zona metropolitana y no sólo los empleos de la ciudad interior. La distancia entre municipios se calculó con base en la red de transporte en vías primarias entre los centroides de las áreas urbanas de los municipios. Las fuentes de información para 1990 y 2000 fueron los respectivos Censos Generales de Población y Vivienda y los Censos Económicos 1989 y 1999. En los ejercicios siguientes, se replican algunos de los análisis hechos por Cervero, pero en la aplicación a la ZMCM se añaden nuevas formas de análisis de accesibilidad derivadas de mediadas utilizadas previamente.

El primer índice calculado es un índice gravitacional básico que simplemente cuenta el número de empleos por municipio y los pondera por la distancia entre municipios elevada a un coeficiente de fricción de acuerdo con el municipio de referencia (ecuación 1). Este índice se calculó para 1990 y 2000. Por ser un índice relativo, al igual que el resto de los índices que presentaremos, los resultados se muestran estandarizados (véase figura III).

Ecuación 1. Índice de accesibilidad básico ${ }^{3}$

$$
A_{i}=\sum_{j} E_{j} \cdot D_{i j}^{-\lambda}
$$

${ }^{3}$ Tomado de Cervero, et al., (1997). 
donde:

$=$ Número de empleos en el centro de empleo $j$.

$D_{i j}=$ Distancia mediante la red de transporte entre las zonas $i$ y $j$.

$\lambda=$ Coeficiente de impedancia ${ }^{4}$ calculado empíricamente como -0.82 .

Los resultados de este primer análisis muestran que, en términos generales y para el periodo en estudio, la ciudad interior tuvo la mayor accesibilidad a empleos. Asimismo, a medida que la distancia al centro aumenta, la accesibilidad decrece. Esto no debería sorprender a nadie. De acuerdo con esta perspectiva, las delegaciones centrales han perdido accesibilidad al igual que los municipios de los contornos tres y cuatro. Por el contrario, las delegaciones y municipios del primer y segundo contornos, han ganado accesibilidad. Esto significa que entre 1990 y 2000, los empleos generados están más cerca de los contornos intermedios y que, por lo tanto, su población ha sido la más beneficiada en cuanto a accesibilidad.

He aquí un problema: ¿qué tan útiles son estos empleos a la población residente? Es decir, ¿̇corresponden a los tipos de empleos que la población de estos municipios y delegaciones requieren?

Para resolver este cuestionamiento se calculó un índice de accesibilidad gravitacional-isocrónico, que es una medida más robusta de accesibilidad. Este índice ajusta las oportunidades de empleos ponderados por el coeficiente de fricción de la distancia, pero en función de distintos sectores económicos, y los pondera por el porcentaje de la PEA municipal que se dedica a cada sector económico. De esta manera, no se sobreestiman las oportunidades de empleo cuando aumentan los empleos de un sector determinado en áreas donde la PEA se dedica a un sector distinto, como sucede con el índice de accesibilidad básico. Los resultados se muestran en las Figuras IV y vII.

\footnotetext{
${ }^{4}$ El coeficiente de impedancia es el resultado de una regresión lineal con transformaciones logarítmicas de las variables número de viajes y distancia calculado a partir de la base de datos de la Encuesta origen-destino 94. El coeficiente representa una elasticidad del porcentaje de reducción de viajes con un incremento de $1 \%$ en el aumento de la distancia. La interpretación de este coeficiente es: qué tan lejos está dispuesta la gente a viajar para trabajar.
} 


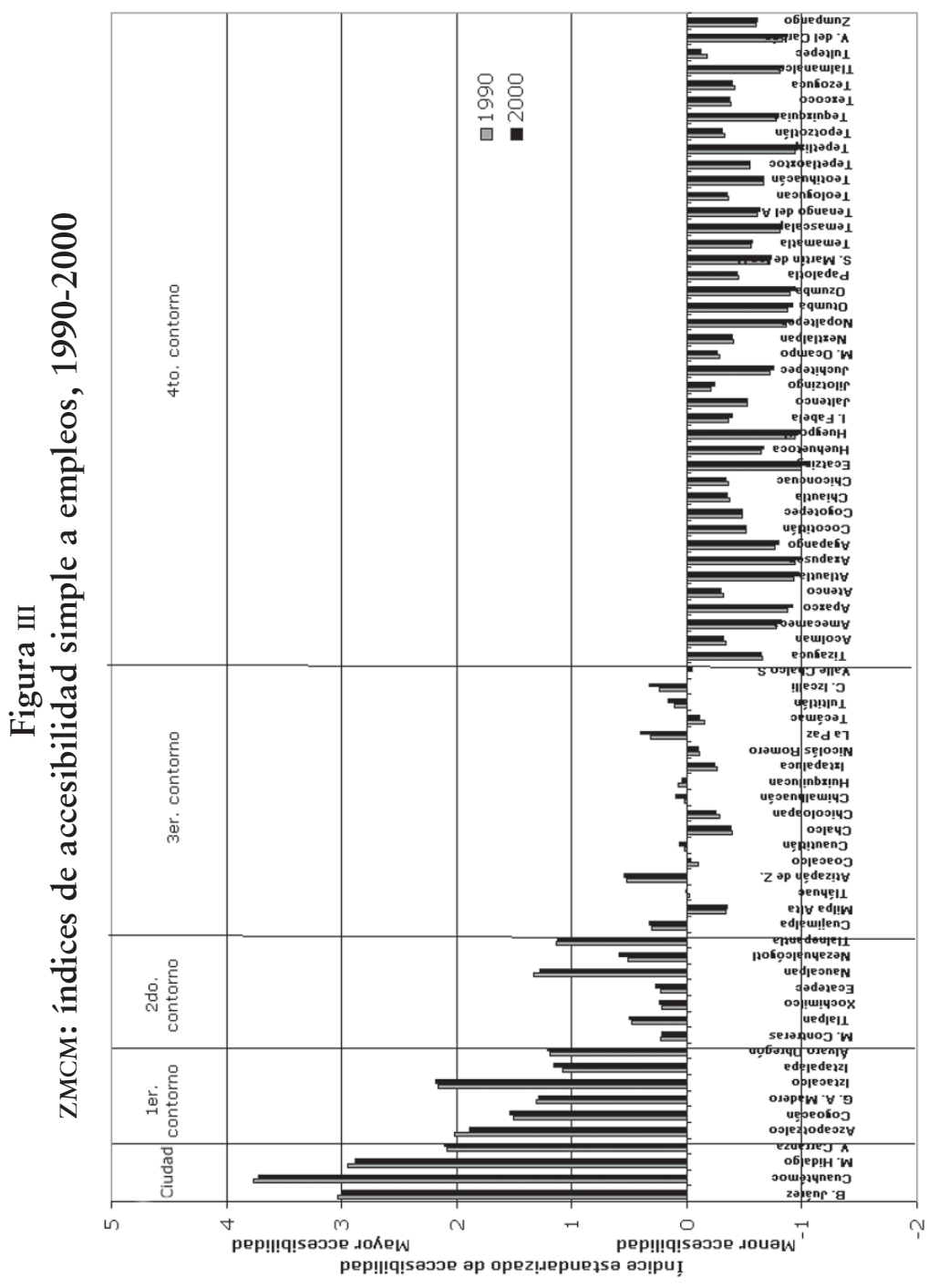


Ecuación 2. Índice de accesibilidad emparejado por sector de empleo ${ }^{5}$

$$
A_{i}=\sum_{i} \sum_{j}\left[p_{i k} \cdot E_{j k}\right] \cdot D_{i j}^{-\lambda}
$$

donde:

$=$ Número de empleos en el sector de ocupación $k$ en el centro de empleo $j$.

$p_{i k}=$ Número de residentes empleados en la zona $i$ en el sector de ocupación $k$.

$D_{i j}=$ Distancia mediante la red de transporte entre las zonas $i \mathrm{y}$ $j$.

$\lambda=$ Coeficiente de impedancia calculado empíricamente $\mathrm{a}$ 0.82 .

$\mathrm{k}=$ Sectores de ocupación ${ }^{6}$ donde $\mathrm{k}=1 \ldots 11$. (1) Minería, (2) Manufacturas, (3) Electricidad y agua, (4) Construcción, (5) Comercio, (6) Transporte, (7) Sector financiero y bienes inmuebles, (8) Servicios profesionales y técnicos (9) Servicios sociales y de salud (10) Servicios personales y, (11) Servicios de restaurantes y hoteles.

Los datos de este segundo análisis muestran resultados casi opuestos a los del modelo simple. Aunque se mantiene el patrón de accesibilidad decreciente centro-periferia, lo relevante en este caso es el cambio de accesibilidad por municipio entre los dos años del periodo (véase figura v). Esta comparación revela que el aumento de empleos en la zona metropolitana, en realidad, generó una mayor accesibilidad en las delegaciones de la ciudad interior, y no que éstas hayan perdido accesibilidad, como se supondría según el índice simple. Por el contrario, las delegaciones centrales ganaron la mayor accesibilidad de toda la zMCM. En esto radica la importancia de saber no sólo cuántos empleos se crean, sino también de qué tipo son, y sobre todo si corresponden a las necesidades y características de la población local.

En los casos del primer y segundo contornos, con la aplicación del índice simple se subestima la accesibilidad, pues no sólo ganaron accesibilidad sino que incluso lo hicieron en mayor cuan-

${ }^{5}$ Tomado de Cervero, et al., (1997).

${ }^{6}$ Se excluyeron los empleos y la PEA de la rama 91 de la Clasificación Mexicana de Actividades y Productos (CMAP), Administración Pública y Defensa. 


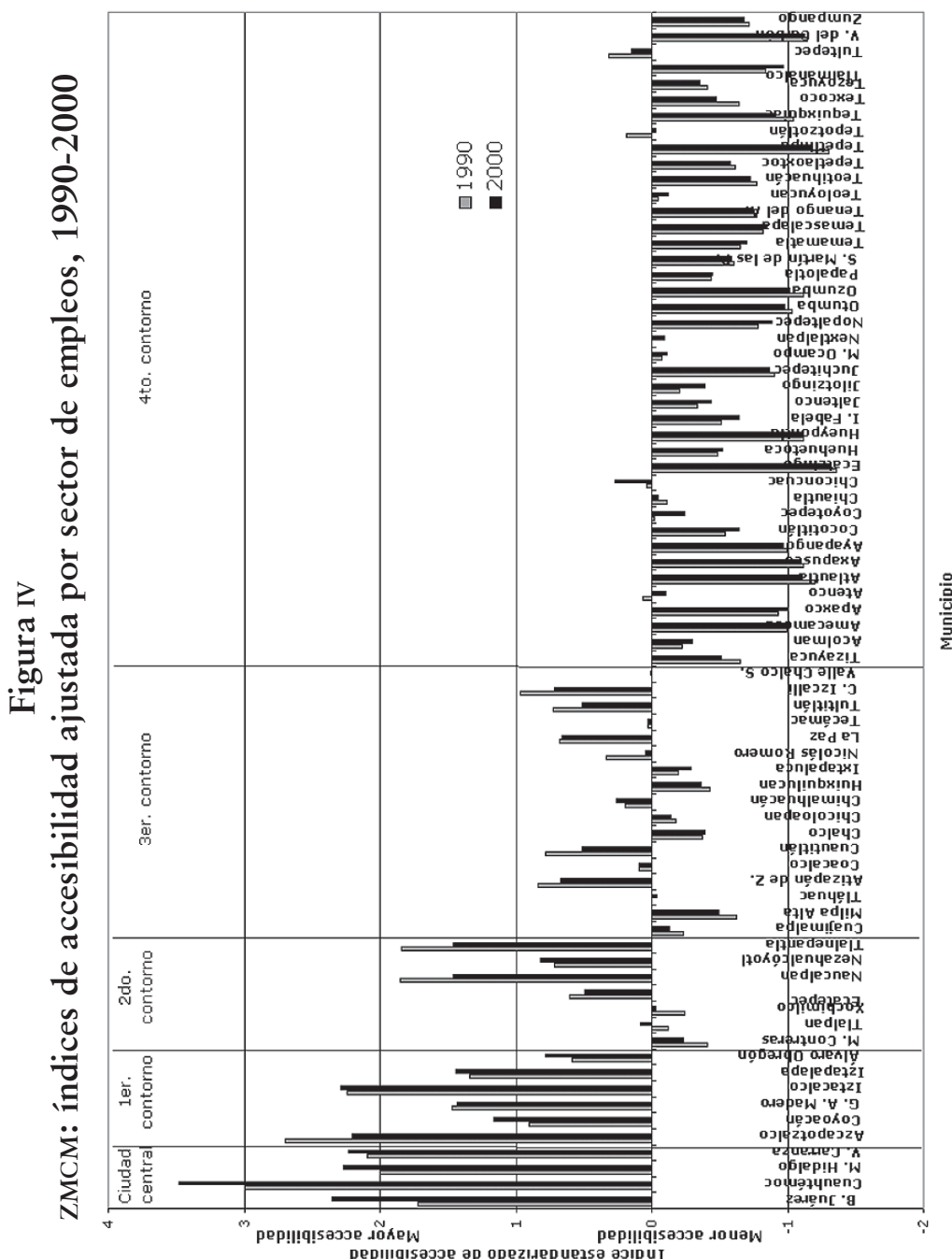




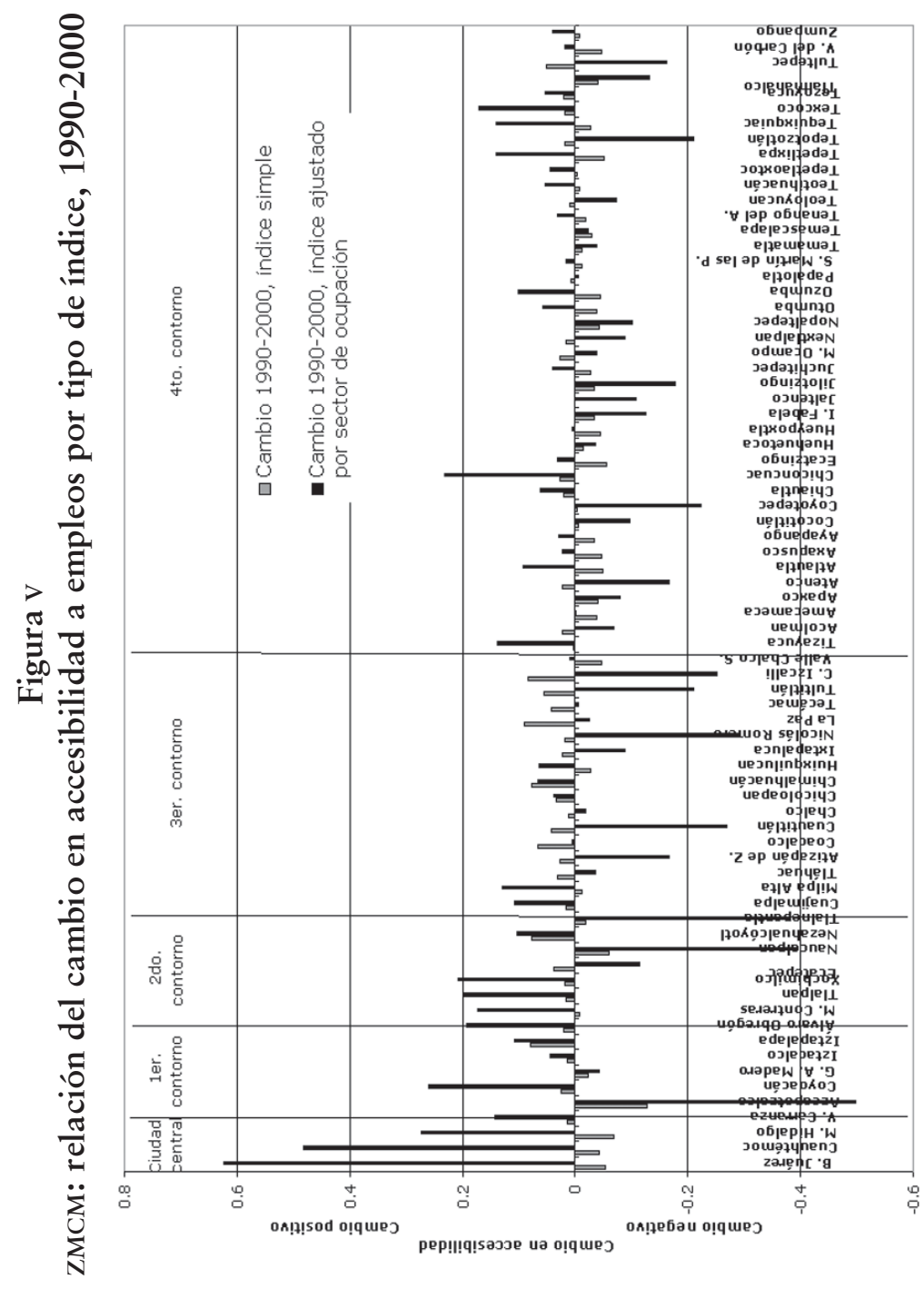

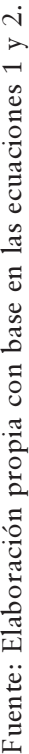


tía. De la misma forma, los que perdieron accesibilidad en realidad pierden más cuando se considera el ajuste por sector de ocupación. En particular son notables los casos de Naucalpan, Tlalnepantla y Ecatepec, comúnmente considerados como centros metropolitanos, ya que, a pesar de estar por encima de la media, han visto disminuir sus índices de accesibilidad.

Para el tercer contorno, con excepción de Cuajimalpa, Milpa Alta y Huixquilucan, el índice simple también sobreestima los cambios de accesibilidad. Al ajustar el índice por sectores de ocupación, en 10 de los 17 municipios de ese contorno disminuye la accesibilidad, y no aumenta, como lo mostraba el primer índice. Finalmente, en el cuarto contorno la variación entre índices es similar a la del primer y segundo contornos. La mitad de los municipios perdieron accesibilidad, mientras que la otra mitad, ganó. Sin embargo, los municipios que perdieron accesibilidad, perdieron en promedio más que la que ganaron el resto de los municipios.

Pero he aquí otro problema. Si bien el índice anterior ajusta los empleos con la PEA por sector de ocupación, aún queda por resolver el efecto que las distintas categorías de ingreso dentro de los distintos sectores económicos pueden tener sobre la accesibilidad. Por ejemplo, en el caso de dos personas que se dedican al sector financiero, cacaso puede considerarse equivalente el empleo que tiene un cajero con el de un vicepresidente corporativo? Es claro que la importancia del ingreso es definitiva. Para captar los diferentes estratos dentro de cada sector se debe modificar la ecuación 2 de la siguiente forma:

Ecuación 3. Índice de accesibilidad ajustado por sector de ocupación y categoría de ingreso ${ }^{7}$

$$
A_{i q}=\sum_{i} \sum_{j}\left[p_{i k q} \cdot E_{j k q}\right] \cdot D_{i j}^{-\lambda}
$$

Las variables son las mismas que en que en la ecuación 2, con las siguientes diferencias:

$E_{j k q}=$ Número de empleos en el centro de empleo $j$, en el sector de ocupación $k$ y en la categoría de ingreso $q$.

$\mathrm{p}_{i k q}=$ Proporción de residentes empleados en la zona $i$ en el sector de ocupación $k$ y en la categoría de ingreso $q$. 


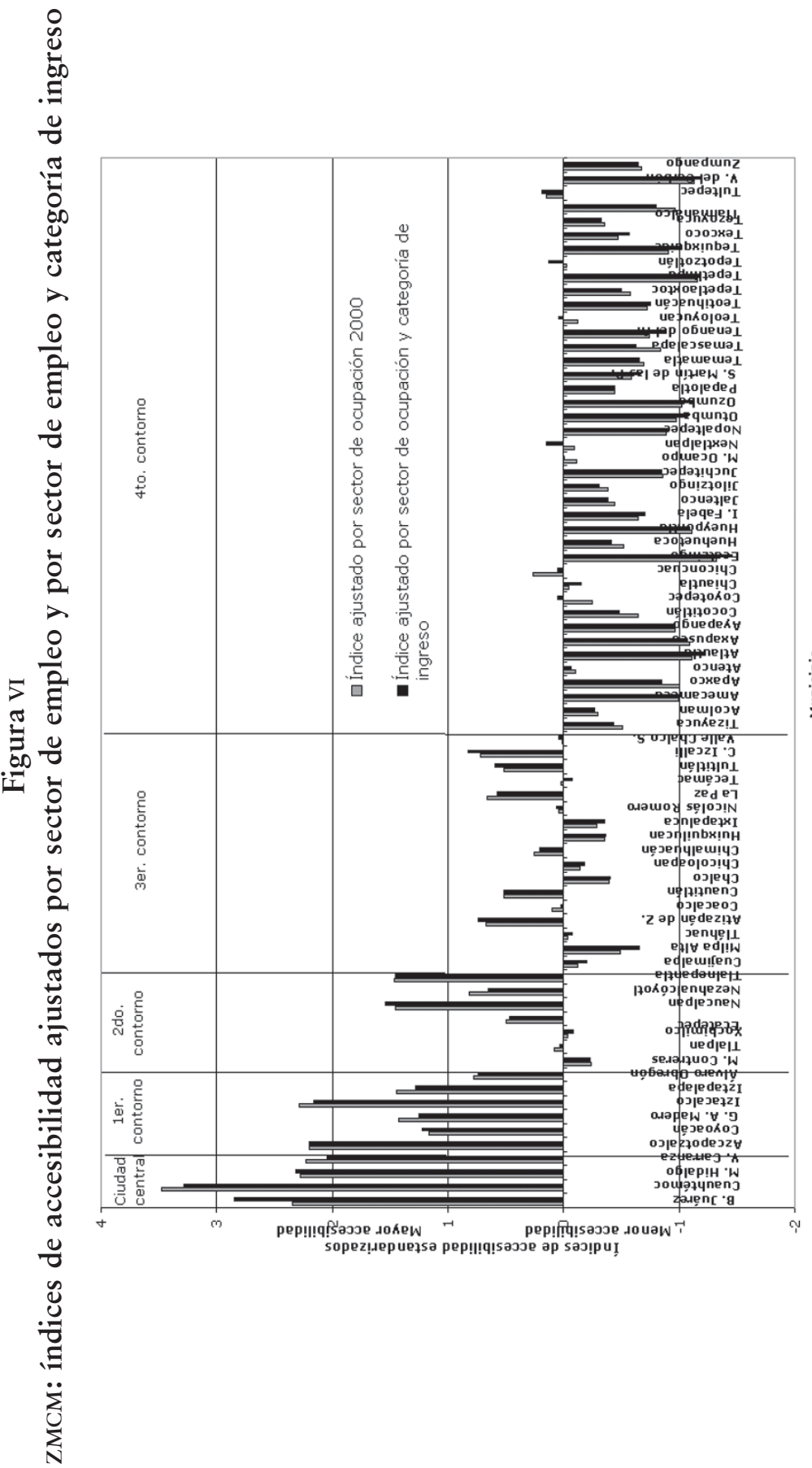


$\mathrm{q}=$ Categorías de ingreso (cuartiles), donde $\mathrm{n}=1$ (Ingreso bajo), 2 (Ingreso medio bajo), 3 (Ingreso medio alto), y 4 (Ingreso alto).

En el cálculo de este tercer índice existe un problema metodológico, pues el Censo Económico sólo muestra las remuneraciones medias por subsector y no el número de empleados por categoría de ingreso. Aunque para 1999 es posible estimar las proporciones de empleos por sector y categoría de ingreso utilizando las variables de ingreso, municipio de trabajo y sector de trabajo del Censo General de Población y Vivienda de 2000, hacer lo mismo para 1989 resulta imposible. Por ello, no es factible hacer ahora una comparación del tercer índice 1990-2000 y habrá que esperar al 2010 para realizar de manera completa este análisis. Sin embargo, si se calcula este último índice para 2000 (véase figura vI), se nota una deficiencia con el índice ajustado solamente por sectores, aunque no tan grande como la diferencia entre este último y el índice simple. Lo más notorio de la comparación entre estos índices es que, en promedio, el índice ajustado solamente por empleos, subestima ligeramente la accesibilidad de los municipios del cuarto contorno y sobreestima, en promedio, la accesibilidad del resto de los contornos, con la notoria excepción de Benito Juárez. Aun así, el patrón de accesibilidad decreciente centro-periferia permanece (véase figura viII).

\subsection{Accesibilidad entre sectores de ocupación y categorías de ingreso}

Se consideró medir la accesibilidad generalizada para la ZMCM y dividirla por sectores de ocupación y categorías de ingreso. Ambos índices reflejan qué estratos de la PEA de acuerdo con su sector de ocupación e ingreso, gozan de mayor accesibilidad.

En este caso, las fórmulas suman la accesibilidad por municipio por medio de los sectores ponderándola por la proporción de la PEA total que reside en cada municipio y que se encuentra dentro del sector, así como por el número de trabajadores en el mismo estrato en cuestión (de otra forma, la accesibilidad sería mayor dependiendo del tamaño del estrato). Los resultados se muestran en las figuras VII y VIII. 
Figura VII

ZMCM: cambio en accesibilidad a empleos ajustada por sector de ocupación, 1990-2000

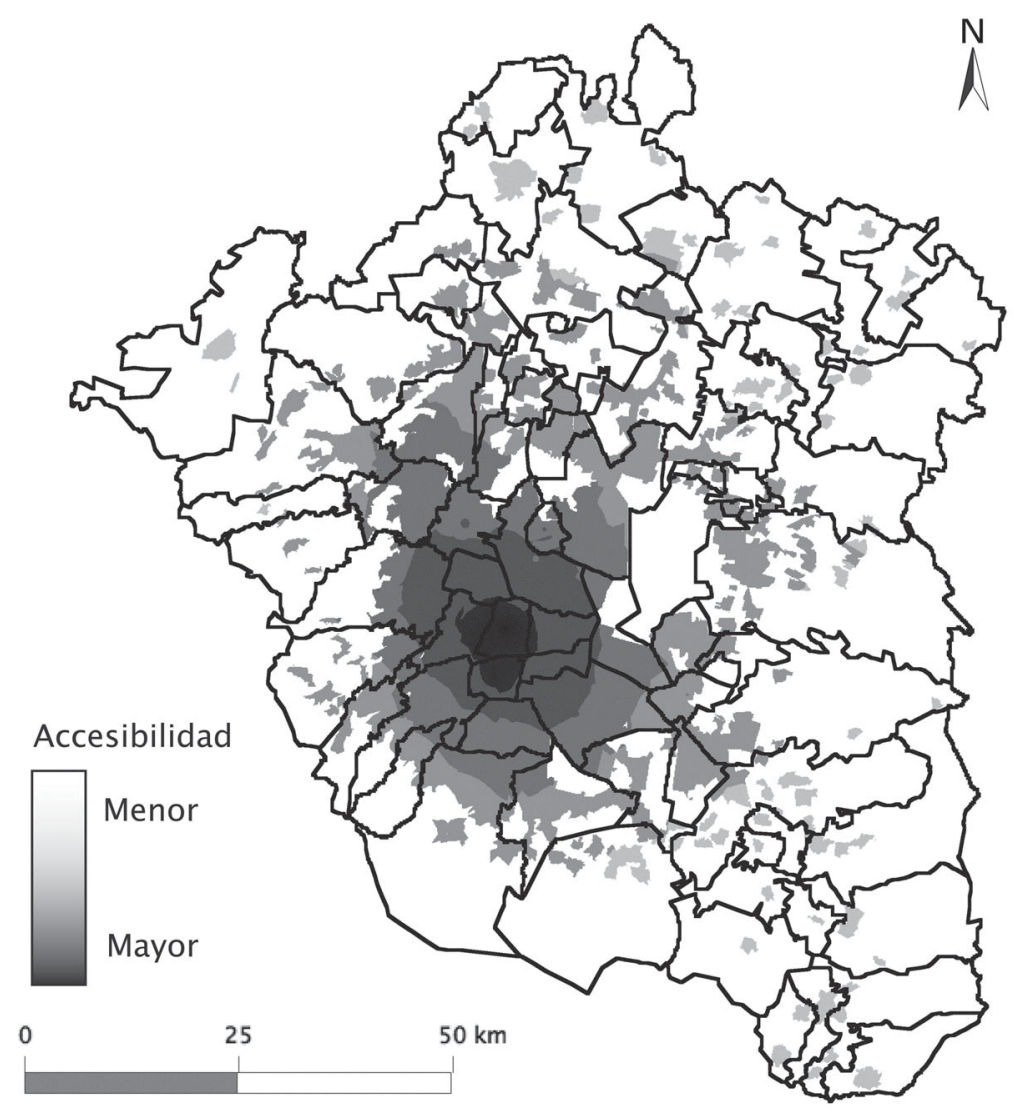

Fuente: Elaboración propia con información del gráfico IV 


\section{Figura VIII}

ZMCM: Accesibilidad a empleos ajustada por sector de ocupación y categoría de ingreso, 2000

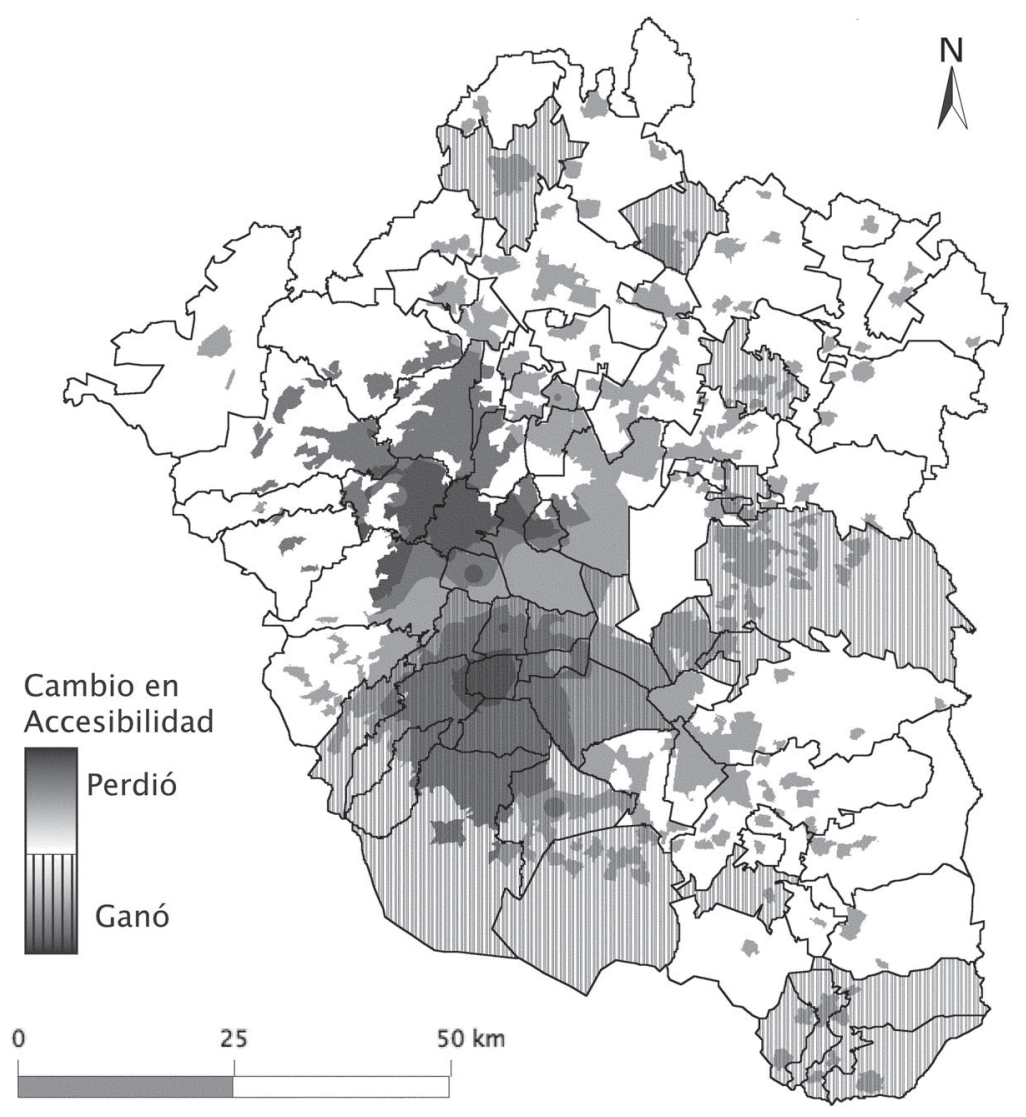

Fuente: Elaboración propia con información del gráfico VI 
Ecuación 4. Accesibilidad entre sectores de ocupación ${ }^{8}$

$$
A_{k}=\sum_{i} \sum_{j}\left[p_{k i} \cdot \frac{E_{j k}}{P_{i k}}\right] \cdot D_{i j}^{-\lambda}
$$

donde:

$E_{j k}=$ Número de empleos en el sector de ocupación $k$ en el centro de empleo $j$.

$p_{k i}=$ Proporción de la PEA ocupada en el sector de ocupación $k$ que reside en la zona $i$.

$P_{i k}=$ Número de residentes empleados en la zona $i$ en el sector de ocupación $k$.

Ecuación 5. Accesibilidad entre categorías de ingreso 9

$$
A_{q}=\sum_{k} \sum_{j}\left[p_{q k i} \cdot \frac{E_{j k q}}{P_{i k q}}\right] \cdot D_{i j}^{-\lambda}
$$

donde:

$p_{q k i}=$ Proporción de la PEA ocupada en el sector de ocupación $k$ y en la categoría de ingreso $q$ que reside en la zona $i$.

$E_{j k q}=$ Número de empleos en el sector de ocupación $k$ y en la categoría de ingreso $q$ en el centro de empleo $j$.

$P_{i k q}=$ Número de residentes empleados en la zona $i$ en el sector de ocupación $k$ y en la categoría de ingreso $q$.

Los resultados del análisis por sector económico (figura Ix) muestran una disminución en la accesibilidad de la PEA dedicada a los sectores manufacturas y construcción (los más grandes del sector secundario), una disminución de accesibilidad en el sector comercio, y variaciones en la accesibilidad de los subsectores de servicios entre 1990 y 2000. En promedio, existe un aumento en la accesibilidad en los servicios, aunque sólo el sector financiero y los servicios profesionales y técnicos (que disminuyeron en accesibilidad) están por encima de la media en el 2000, junto con el pequeño sector electricidad y agua.

${ }^{8}$ Ajustada a partir de Cervero (1997). La fórmula original no considera el ajuste por el número de residentes ocupados en el sector, y no es claro si multiplica por la proporción del sector que reside en el municipio o si lo hace por la proporción de la PEA del municipio dedicada al sector.

${ }^{9}$ Derivada a de la ecuación 4 . 
Figura IX ZMCM: accesibilidad entre sectores de empleo, 2000

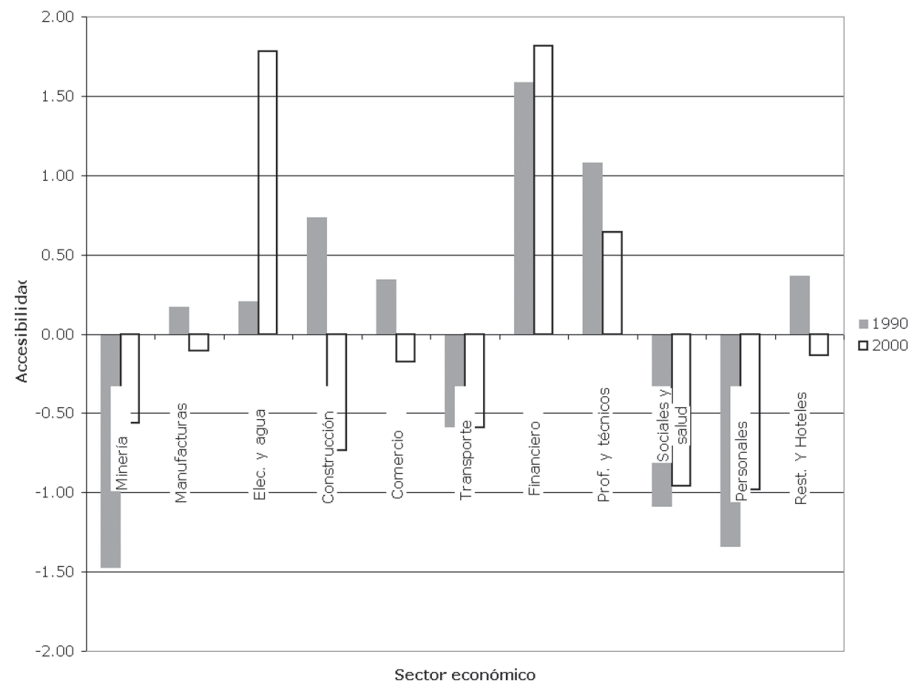

Fuente: Elaboración propia con base en la ecuación 4.

Figura $\mathrm{X}$

ZMCM: accesibilidad entre categorías de ingreso, 2000

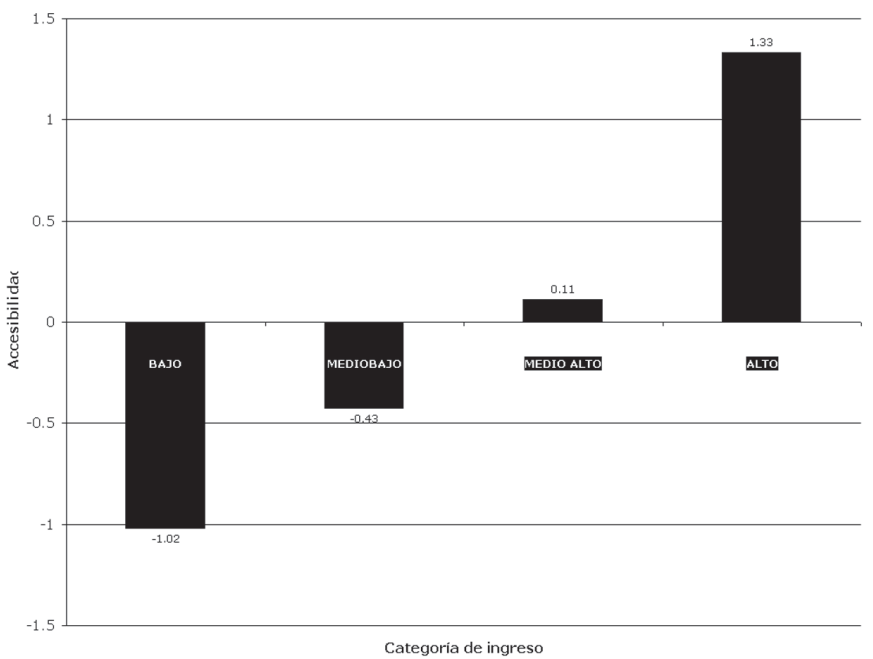

Fuente: Elaboración propia con base en la ecuación 5. 
Para el caso de la accesibilidad por categoría de ingreso (véase figura $\mathrm{x}$ ), se encuentra un patrón que, si bien resulta lógico, es alarmante. Dentro de los respectivos sectores de ocupación (dada la ecuación 5) la mitad de la PEA con ingresos medios bajos y bajos se encuentra por debajo de la media de accesibilidad metropolitana, mientras que la PEA de las categorías de ingreso medio alto y alto se encuentran por encima de la media de accesibilidad. Esto significa que la PEA de ingresos altos goza de una accesibilidad 160\% mayor a la de ingresos bajos, lo que, sin duda, refleja una profunda segregación social derivada del ingreso de las familias. Más aún si se considera que las familias de altos ingresos pueden elegir un lugar de alta o baja accesibilidad para su residencia, mientras que los de escasos ingresos no pueden hacerlo.

\section{2. ¿Planeación con base en accesibilidad?}

La planeación del transporte urbano con base en la movilidad ha sido la forma predominante del desarrollo de la infraestructura de transporte. Los ejes viales, los grandes pasos a desnivel, segundos pisos y avenidas de seis o más carriles son claros ejemplos de ello. En cambio, la planeación basada en la accesibilidad toma en cuenta no sólo la velocidad y capacidad de desplazamiento: combina la planeación de la infraestructura con la de los usos del suelo y acerca las actividades, reduciendo la fricción de la distancia.

En esta sección se muestran una serie de análisis de regresión que muestran cómo los municipios que cuentan con una mayor proporción de trabajadores provenientes de zonas de mayor accesibilidad, tienen una mayor producción per cápita que aquellos en áreas de menor accesibilidad, lo que permite concluir que la accesibilidad no es simplemente un indicador de calidad de vida sino también un insumo para la productividad de la ciudad. Posteriormente se presentan otros dos modelos que permiten identificar los factores que contribuyen a generar zonas de alta accesibilidad, con la intención de mostrar que se pueden elaborar políticas públicas con estos factores en mente.

Para determinar si la accesibilidad es simplemente un índice de medición de oportunidades de empleo o si, en efecto, puede utilizarse como un criterio de formulación de política pública, se calculó la accesibilidad de los municipios y delegaciones metropolitanas, pero no de la que gozan los residentes sino la de quie- 
nes trabajan en cada municipio. Para ello se utilizó la base de datos del censo del 2000 y se extrajo la proporción de trabajadores por municipio de acuerdo con su municipio de residencia. Utilizando la ecuación 6, se calculó el índice de accesibilidad para zonas de empleo.

Ecuación 6. Accesibilidad entre centros de empleo

$$
A_{j}=\sum_{i} A_{i} \cdot p_{i j}
$$

donde:

= Accesibilidad empatada por sector de ocupación e ingreso en la zona residencial $i$.

$p_{i j}=$ Proporción de empleados del centro de empleo $j$ que residen en la zona residencial $i$.

Posteriormente, este índice se utilizó como variable en un modelo de regresión lineal cuya variable dependiente es el valor de producción bruta per cápita. El modelo se corrió innumerables veces con distintos grupos de variables predictivas de carácter urbano. En el cuadro 1 se muestra el modelo más robusto que se generó.

El primer resultado relevante de este análisis de regresión es que los dos factores que más aportan a la productividad per cápita son: la educación, y el hecho de que los trabajadores de los centros de empleo provengan de zonas de alta accesibilidad. Ambas variables afectan positivamente a la producción. Es inte-

\section{Cuadro 1}

Regresión lineal: producción per cápita, ZMCM 2000

\begin{tabular}{lccr}
\hline & Coeficientes & $\begin{array}{c}\text { Error } \\
\text { estándar }\end{array}$ & Beta \\
\hline (Constante) & -93.4 & 155.8 \\
Accesibilidad en centro de empleo & 54.9 & 18.4 & 0.4 \\
Escolaridad media de los trabajadores & 54.6 & 16.7 & 0.43 \\
$\quad$ del municipio & & & \\
Densidad de empleo & -0.4 & 0.5 & -0.09 \\
Densidad de PEA & -2.2 & 0.7 & -0.34 \\
CL comercio & -93.6 & 21.1 & -0.35 \\
Proporción PEA/empleos & -2.3 & 2.2 & -0.08 \\
$\mathrm{R}^{2}$ ajustada = 0.681 & $\mathrm{F}=25.8$ & Sig. 000 & \\
\hline
\end{tabular}

Fuente: Elaboración propia con base en cálculos anteriores y en INEGI (1999 y 2000). 


\section{Cuadro 2 \\ Regresión lineal: Accesibilidad en zonas de empleo ZMCM, 2000}

\begin{tabular}{lrrr}
\hline & Coeficientes & $\begin{array}{c}\text { Error } \\
\text { estandar }\end{array}$ & Beta \\
\hline (Constante) & 184.2 & 3095.3 & \\
Accesibilidad municipal & 4049.5 & 193.1 & 1.122 \\
Densidad de empleos & -46.5 & 6.1 & -0.343 \\
Densidad de PEA & 15.6 & 7.2 & 0.088 \\
IVC Ingreso & 11027.8 & 3504.8 & 0.093 \\
$\mathrm{R}^{2}$ ajustada $=0.978$ & $\mathrm{~F}=353$ & Sig. 000 & \\
\hline
\end{tabular}

Fuente: Elaboración propia con base en cálculos propios y en INEGI (1999 y 2000).

resante que la educación, a juzgar por los coeficientes estandarizados (Beta), explica sólo 7\% más de la variación en la producción que la accesibilidad. El modelo muestra que, en municipios especializados en comercio y donde la PEA rebasa al número de empleos, la productividad per cápita disminuye. Por otro lado, la densidad de empleos y la densidad de la PEA muestran coeficientes negativos, lo que es, sin duda, contraintuitivo. Este extraño comportamiento de las variables se explicará más adelante, en el tercer análisis.

El segundo análisis consistió en observar qué variables tenían el mayor efecto explicativo en la accesibilidad a centros de empleo. Las variables utilizadas en el modelo fueron el índice de accesibilidad municipal, la densidad de empleos y de PEA, y un índice de variación cualitativa de ingreso. Este índice muestra qué tan homogéneo o heterogéneo es un municipio en cuanto a la composición de estratos económicos de la PEA. A medida que aumenta el índice, el municipio es más heterogéneo; es decir, las categorías de ingreso están más equitativamente representadas.

La variable que más contribuye a la accesibilidad de la zona de empleo es, precisamente, el hecho de que el municipio sea accesible en términos de sus residentes. Esto, si bien lógico, es importante. Si la PEA y sus categorías están ajustadas con los tipos de empleo cercanos, los residentes tenderán a trabajar cerca de sus hogares ya sea en el municipio de residencia o en municipios cercanos, lo que se traduce en mayor productividad per cápita. En este caso, la densidad de PEA muestra un coeficiente positivo, aunque la densidad de empleos aún es negativa. Finalmente, el modelo muestra que las zonas con mayor heterogeneidad de ingreso; es decir, de ingresos mixtos, tienden a ser 


\section{Cuadro 3}

Regresion lineal: accesibilidad por municipio (empatada por sector de ocupación y categoría de ingreso), ZMCM 2000

\begin{tabular}{lccc}
\hline & Coeficientes & $\begin{array}{c}\text { Error } \\
\text { estándar }\end{array}$ & Beta \\
\hline (Constante) & -3.889 & 1.8 & \\
IQVINC00 & 3.516 & 2.1 & 0.108 \\
JOBDEN00 & $1.92 \mathrm{E}-02$ & 0 & 0.497 \\
PEADEN00 & $2.02 \mathrm{E}-02$ & 0 & 0.401 \\
$\mathrm{R}^{2}$ ajustada $=0.751$ & $\mathrm{~F}=74$ & Sig. 000 & \\
\hline
\end{tabular}

Fuente: Elaboración propia con base en cálculos propios y en INEGI (1999 y 2000).

zonas de empleo de mayor accesibilidad. Aun con una $\mathrm{R}^{2} \tan$ elevada, no se encontraron, en este análisis, problemas de heteroscedasticidad ni de covariación a juzgar por el análisis de residuos y los índices de tolerancia.

Hasta aquí se ha dicho que la accesibilidad de los centros de empleo explica gran parte del aumento en la producción per cápita de los trabajadores y que la accesibilidad municipal (residentes) explica la mayor parte de la accesibilidad en los centros de empleo, pero ¿qué explica la accesibilidad municipal? En el cuadro 3 se muestra que tres variables explican $75 \%$ de la varia-

\section{Figura XI}

Modelo causal de densidad de empleo, PEA, accesibilidad y producción per cápita

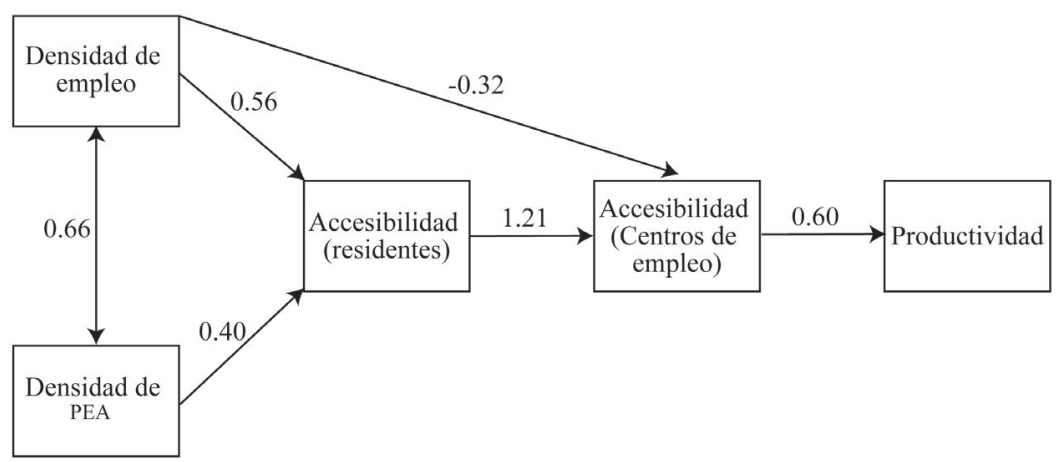

Fuente: Elaboración propia. 
ción en la accesibilidad municipal: la densidad de empleos, la densidad de la PEA, y la mezcla de ingresos.

Las tres variables utilizadas afectan de manera positiva la accesibilidad, que, a su vez, afecta de manera positiva la accesibilidad en centros de empleo y que, a su vez, tiene un efecto positivo en la productividad. Esto explica por qué la densidad de empleos y PEA mostraban coeficientes negativos en los análisis anteriores. Es decir, las densidades actúan de manera positiva sobre la producción, pero por medio de la accesibilidad. La forma simplificada del modelo causal se muestra en la figura XI.

\section{Conclusiones}

En resumen, de acuerdo con el índice de accesibilidad ajustado por sectores de ocupación, la suburbanización de empleo y los cambios en la estructura socioeconómica-espacial en la ZMCM entre 1990 y 2000 no fueron eficientes. Los niveles de accesibilidad se han incrementado más en el centro y, en general, disminuyen a medida que aumenta la distancia al mismo. Esto significa que las zonas de mayor crecimiento poblacional entre 1990 y 2000 son las que más carecen de empleos adecuados a las necesidades de sus residentes. Desde esta perspectiva, se fortalece la idea de que la ciudad de México está inmersa en un proceso de monocentrismo disperso y no en uno de policentrismo.

Del cálculo de la accesibilidad por sector económico y categorías de ingreso se deriva que existe una alta segregación socioeconómica-espacial, y que, en las categorías más bajas de ingreso se tienen menores oportunidades de empleo en cercanía al lugar de residencia. De esto se puede deducir que quienes son pobres se ven obligados a pagar una alta proporción de sus ingresos, tal vez no en vivienda económica construida en la periferia, pero sí en transporte para encontrar empleo formal. Adicionalmente, se demostró que las zonas con mayor accesibilidad tienden a tener una producción más alta por trabajador, aun si los niveles de educación de los trabajadores, la proporción de PEA residente y empleos, densidades y especialización económica, son los mismos.

¿Cuáles son entonces las políticas públicas idóneas para impulsar la formación de zonas con mayor accesibilidad a empleos? Existen cuatro alternativas:

El sentido común aconsejaría la descentralización de empleos dentro de la metrópoli. Aunque esta opción es políticamente co- 
rrecta, no deja de ser romántica y de pocos efectos útiles. Si 40\% de los empleos se concentran en la ciudad interior, que representa menos de 7\% del área urbana, esto debe ser por algo. Las teorías generales de localización, de aglomeración, de causación acumulativa (cumulative causation) o retroalimentación positiva (positive feedbacks), incluso las ideas de capital social e interacción cara a cara (face to face interaction), lo explican. Dado esto, ¿qué tipo de ventajas de localización podría ofrecer un municipio del cuarto contorno metropolitano a una empresa, que no sea el bajo precio del suelo, más aún en una ciudad que tiende a la terciarización? Efectivamente, los precios altos del suelo en la zona central se justifican por el aumento de productividad que esta zona tiene, junto con el ahorro en transporte. Entonces, ¿qué tipos de empleo podrían atraer los municipios periféricos? Si, además, los 75 municipios y delegaciones de la zona metropolitana están en competencia por atraer empleos, ¿qué tipos de densidades se podrían generar?, ¿qué asegura que los empleos serían apropiados para los residentes de un municipio si las empresas se dispersan por todo el territorio metropolitano?, ¿no generaría esto mayor expansión y desbordamiento, además de requerir una mayor infraestructura de transporte para satisfacer la demanda por nuevos flujos de transporte metropolitanos? La evidencia presentada en este ensayo muestra que se requiere de la concentración de empleos y población, no la dispersión de éstos.

La segunda alternativa se basa en la justificación teórica del monocentrismo, esto es, que el centro de una ciudad es, en promedio, el punto más cercano a cualquier otro. Esta opción es contraria a la primera y consiste en elevar la oferta de vivienda en cercanía a las áreas de empleo existentes. Para lograrlo se requiere de políticas de densificación residencial de acuerdo con el supuesto económico de que, a mayor oferta, los precios de la vivienda tienden a reducirse. ${ }^{10}$ Esta política tendría que ir acompañada de una zonificación de usos mixtos e implica intensificar aún más la actividad económica y residencial del centro. Una política de este tipo requiere de una infraestructura de transporte masivo eficiente (Metro y Metrobús) que pueda absorber el congestionamiento que produciría una elevación de densidades. La gran desventaja de esta alternativa es el alto costo social que implica, por la probable resistencia de los residentes locales, como sucede actualmente.

${ }^{10}$ Esto es, en efecto, un fenómeno actualmente observable en diversos estados en donde existe una sobreoferta de vivienda de interés social, aunque no en áreas urbanas centrales. 
La tercera alternativa es una combinación de las dos anteriores. Implica encaminar la creación de empleos y vivienda en densidades eficientes en lugares seleccionados del área metropolitana y que cuenten con conexiones con el centro por medio de transporte eficiente. Es decir, una política de subcentros alrededor de nodos de transporte. Ésa debiera ser la alternativa más eficiente de acuerdo con la teoría existente. Sin embargo, la experiencia que hasta el momento ha brindado para la ciudad, Santa Fe, ha sido menos que recompensante. De acuerdo con los Censos Económicos de 1999, el número de empleos en la zona planeada de Santa Fe aún no ascendía a 10,000 empleos (de unos cuatro millones en el área metropolitana). Por supuesto, ésta es un área de poco acceso al transporte público, y de débil interconexión entre usos residenciales, comercio y oficinas. Es un área de usos no mixtos sino adyacentes-segregados y que no ha logrado ser un subcentro contenido, quizá, por deficiencias en su planeación. Finalmente, como alguna vez sugirió Richardson con ironía, la cuarta alternativa es no hacer nada. Dejar al mercado actuar por sí mismo y permitir que la ciudad siga el rumbo que ha tomado.

La aportación más importante del presente análisis ha sido comprobar cuantitativamente que sí existe un desequilibrio espacial que se acentuó entre 1990 y 2000 y que en efecto se trata, para el caso de Ciudad de México, de una falta de equilibrio entre empleo y vivienda y de áreas compactas y conectadas entre sí. Los análisis de regresión y el modelo causal aquí presentados, muestran que una mayor accesibilidad se puede obtener al elevar las densidades de empleo y residenciales locales combinando categorías de ingreso entre los residentes. De ello se deriva que las políticas de transporte basadas en la movilidad tendrán poco efecto en la accesibilidad si no se prevén de manera conjunta el transporte y el uso del suelo urbano. Dejar al mercado actuar, más allá de su justa medida, representa el mejor de los mundos posibles para el capital, pero difícilmente se puede hacer pasar como ejemplo de una política metropolitana encomiable para el conjunto social, sobre todo de los sectores de la población más desafortunados.

\section{Bibliografía}

Aguilar, Adrián Guillermo y Concepción Alvarado (2005), "La reestructuración del espacio urbano de la Ciudad de México. ¿Hacia la metrópoli multinodal?”, en Adrián Guillermo Aguilar (coord.), Procesos metropolitanos y grandes 
ciudades. Dinámicas recientes en México y otros países, Porrúa, México, pp. 265-308.

Alonso, William (1964), Location and Land Use, MIT Press, Boston.

Arnott, Richard (1997), Economic Theory and the Spatial Mismatch Hypothesis, Boston College, Working Papers in Economics 390.

Cervero, Robert (1989), “Jobs-Housing Balance and Regional Mobility", Journal of the American Planning Association, 55: 136-150.

Cervero, Robert (1995), Polycentrism, Commuting, and Residential Llocation in the San Francisco Bay Area, University of California at Berkeley, Institute of Urban and Regional Development, Working Paper 640.

Cervero, Robert (1996), “Jobs-Housing Balance Revisited. Trends and Impacts from the San Francisco Bay Area", Journal of the American Planning Association, 62 (4): 492-511.

Cervero, Robert (1997), “Tracking Accessibility", Access, (11): 27-31.

Cervero, Robert, Timothy Rood y Bruce Appleyard (1997), Job Accessibility as a Performance Indicador: An Análisis of Trends and their Social Policy Implications in the San Francisco Bay Area, IURD, Working Paper 692.

Cruz-Rodríguez, María Soledad (2001), Propiedad, poblamiento y periferia rural en la Zona Metropolitana de la Ciudad de México, Programa Editorial de la Red de Investigación Urbana-Universidad Autónoma Metropolitana, Unidad Azcapotzalco, Puebla y México, Distrito Federal.

Chapple, Karen (2001), “Time to work: Job search strategies and commute time for women on welfare in San Francisco", Journal of Urban Affairs 23 (2): 155-173.

Delgado, Javier; Patricia Ramírez; Mario Salgado y Margarita Camarena (1999), "Estructura metropolitana y transpor- 
te", en Roberto Eibenschutz (coord.), Bases para la planeación del desarrollo urbano en la Ciudad de México, Porrúa, México, pp. 7-67.

Giuliano, Genevieve y Kenneth A. Small (1993), “Is The journey to Work Explained by Urban Structure?" Urban Studies 30 (9): 1485-1500.

Graizbord-Ed, Boris y Beatriz Acuña (2005), "La estructura polinuclear del Área Metropolitana”, en Adrián Guillermo Aguilar (coord.), Procesos metropolitanos y grandes ciudades. Dinámicas recientes en México y otros países, Porrúa, México, pp. 309-328.

Handy, Susan (1993), "Regional Versus Local Accessibility: Implications for Nonwork Travel", Transportation Research Record, (1400): 58-66.

Holzer, Harry J.; Keith Ihlanfeldt R. y David Sjoquist L. (1994), "Work, Search and Travel among White and Black youth", Journal of Urban Economics, 35: 320-345.

Ihlanfeldt, Keith y David Sjoquist (1989), "The impact of decentralization on the economic welfare of central city blacks", Journal of Urban Economics 26: 110-130.

INEGI (1989), Censos económicos 1988, Instituto Nacional de Estadística Geografía e Informática, Aguascalientes.

INEGI (1990), XI Censo de población y vivienda 1990, Instituto Nacional de Estadística Geografía e Informática, Aguascalientes.

INEGI (1999), Censos económicos 1998, Instituto Nacional de Estadística Geografía e Informática, Aguascalientes.

INEGI (2000), XII Censo de población y vivienda 2000, Base de datos de la muestra, Instituto Nacional de Estadística Geografía e Informática, México.

Kain, John (1994), “The Spatial Mismatch Hypothesis: Three Decades Later”, Housing Policy Debate, 3: 371-462. 
Lizt, Sonia (1988), "Respuestas del transporte urbano en las zonas marginadas", en Raúl Benítez y José B. Morelos (coords.), Grandes problemas de la Ciudad de México, Plaza y Valdés, México, pp. 215-242.

Mills, Edwin S. (1972), Studies in the Structure of the Urban Economy, The Johns Hopkins Press, Baltimore.

Muñoz, Gerardo (1995), "Expectativas del transporte en la megalópolis de la ciudad de México", en Luis Chías (coord.), El transporte metropolitano hoy, UnAm, Mexico, pp. 97108.

Murata, Masanori; Manuel Suárez; y Javier Delgado (2005), "Why do the Poor Travel Less? Urban Structure, Commuting and Economic Informality in Mexico City", trabajo presentado en el Congreso de la Comisión Urbana de la Unión Geográfica Internacional, Tokio.

Navarro, Bernardo (1988), "El transporte de la fuerza laboral”, en Raúl Benítez y José B. Morelos, (coords.), Grandes problemas de la Ciudad de México, Plaza y Valdés, México, pp. 243-264.

O’Regan, Katherine M. y John M Quigley (1993), “Family Networks and Youth Access to Jobs", Journal of Urban Economics, 34: 230-248.

Quintanilla, Juan (1995), "Reordenación de la jornada laboral urbana como una medida de manejo de la demanda en el transporte, uso racional de la energía y reducción de emisiones en la Zмсм", en Luis Chías, (coord.), El transporte metropolitano hoy, unam, México, pp. 85 -96.

Suárez, Manuel y Javier Delgado (en prensa), "La expansión urbana probable de la Ciudad de México. Un escenario pesimista y dos alternativos para el año 2020", Estudios Demográficos y Urbanos, El Colegio de México.

Villavicencio, Judith (1999), "La vivienda en el Distrito Federal: necesidad de cambios en la política habitacional actual", en Roberto Eibenschutz, (coord.), Bases para la planea- 
ción del desarrollo urbano en la Ciudad de México, Porrúa, México, pp. 259-313.

Recibido: 11 de octubre de 2005. Aceptado: 12 de agosto de 2006.

Manuel Suárez-Lastra es maestro en Planeación Urbana y Regional por la Universidad de California, Berkeley y es candidato a doctor en Geografía por la Universidad Nacional Autónoma de México. Su línea de investigación se centra en la estructura urbana y movilidad residencial. Entres sus publicaciones destacan: "La expansión probable de la ZMCM a 2020”, Estudios Demográficos y Urbanos, El Colegio de México, México (en Prensa); "La estructura urbana de la ZMCM: Dinámicas de empleo, vivienda y crecimiento metropolitanos", en Los retos del desarrollo metropolitano, Centro de Estudios Sociales y de Opinión Pública, Cámara de Diputados, México; Mexico City@2020: Urban expansion probabilities en Murayama, Y. y G. Du (eds.), Cities in Global Perspective: Diversity and Transition, College of Tourism, Rikkyo University, IGU Urban Comisión, Tokio.

Javier Delgado-Campos es doctor en urbanismo por la Universidad Nacional Autónoma de México. Es investigador titular A en el Instituto de Geografía de la UNAM. Su línea de investigación es la estructura urbana y desarrollo metropolitano e interfase urbanoregional. Ha participado como dictaminador en diversas publicaciones académicas, tales como Revista Investigaciones Geográficas, Boletin del Instituto de Geografía, Revista Sociedad y Territorio, Revista Sociológica, Revista Anuario de Estudios Urbanos, Revista Mexicana de Sociología, Revista Estudios Urbanos y Demográficos y Revista Espacio, Sociedad y Territorio. Entre sus publicaciones destacan: "La urbanización difusa, arquetipo territorial de la ciudad region", en Revista Sociológica, enero-abril, año 18, núm. 51, ISSN 0187-0173, pp. 13-48; “¿Territorios de la globalización o territorios de la crisis? La desruralización en México y la Región Centro", en coautoría con J. Arellanes, en Collado. C. (coord.), La Ciudad de México, Historia y perspectiva, tomo II, pp. 378401, Instituto Mora, México. 